\title{
Tedizolid phosphate for the treatment of acute bacterial skin and skin-structure infections: an evidence-based review of its place in therapy
}

This article was published in the following Dove Press journal: Core Evidence

\author{
Matteo Bassetti ${ }^{1,2}$ \\ Nadia Castaldo' \\ Alessia Carnelutti' \\ Maddalena Peghin' \\ Daniele Roberto Giacobbe ${ }^{2}$ \\ 'Infectious Diseases Division, \\ Department of Medicine, University of \\ Udine and Azienda Sanitaria Universitaria \\ Integrata di Udine, Udine, Italy; \\ ${ }^{2}$ Department of Health Sciences, \\ University of Genoa, Genoa, Italy
}

\begin{abstract}
Introduction: Tedizolid phosphate is an oxazolidinone approved for the treatment of acute bacterial skin and skin-structure infections (ABSSSIs) and active against methicillin-resistant Staphylococcus aureus.
\end{abstract}

Aims: The objective of this article was to review the evidence for the efficacy and safety of tedizolid phosphate for the treatment of ABSSSI.

Evidence review: Approval of tedizolid phosphate for the treatment of ABSSSI was based on the results of two phase III randomized controlled trials, ESTABLISH-1 (NCT01170221) and ESTABLISH-2 (NCT01421511), comparing 6-day once-daily tedizolid vs 10-day twicedaily linezolid. In ESTABLISH-1, noninferiority was met with early clinical response rates of $79.5 \%$ and $79.4 \%$ in tedizolid and linezolid groups, respectively (difference $0.1 \%, 95 \% \mathrm{CI}$ $-6.1 \%$ to $6.2 \%$, with a $10 \%$ noninferiority margin). In ESTABLISH-2, noninferiority was met with $85 \%$ and $83 \%$ rates of early clinical response in tedizolid and linezolid groups, respectively (difference $2.6 \%, 95 \% \mathrm{CI}-3.0 \%$ to $8.2 \%$ ). Pooled data from ESTABLISH-1 and ESTABLISH-2 indicated a lower frequency of thrombocytopenia in tedizolid-treated than in linezolid-treated patients.

Conclusion: Tedizolid offers the option of an intravenous to oral switch, allows once-daily administration, and presents lower risk of myelotoxicity when a 6-day course is used for the treatment of ABSSSI. Greater economic cost associated with this antibiotic could be offset by its shorter treatment duration and possibility of oral administration in routine clinical practice, although either sponsored or nonsponsored postmarketing observational experience remains essential for ultimately confirming the effectiveness and tolerability of tedizolid outside clinical trials.

Keywords: ABSSSI, MRSA, oxazolidinone, Staphylococcus, efficacy, safety

\section{Scope, aims, and objectives}

Acute bacterial skin and skin-structure infections (ABSSSIs) are defined as bacterial infections of the skin with a lesion area of at least $75 \mathrm{~cm}^{2}$. Their clinical presentation is heterogenous, ranging from mild infections to life-threatening invasive diseases. ${ }^{1,2}$ ABSSSIs affect both outpatients and inpatients, and an important increase in ambulatory visits and hospital admissions for ABSSSIs has been observed over the last two decades. ${ }^{3,4}$ This reflects increases in incidence and severity of ABSSSI, relying at least in part on population aging and the related expansion of comorbid conditions, which predispose to either development or worsening of ABSSSI. ${ }^{1,2}$
Correspondence: Matteo Bassett Infectious Diseases Division, Department of Medicine, University of Udine and Azienda Sanitaria, Piazzale Santa Maria della Misericordia, 33100 Udine, Italy

Tel +390432559353

Fax +390432559360

Email matteo.bassetti@asuiud.sanita.fvg.it 
Table I Core evidence clinical impact summary for tedizolid phosphate for the treatment of ABSSSI

\begin{tabular}{|l|l|l|}
\hline Outcome measure & Evidence & Implications \\
\hline $\begin{array}{l}\text { Disease-oriented evi- } \\
\text { dence } \\
\text { Early clinical response }\end{array}$ & $\begin{array}{l}\text { Randomized, double- } \\
\text { blind, noninferiority clini- } \\
\text { cal trial }\end{array}$ & $\begin{array}{l}\text { 6-day once-daily oral tedizolid noninferior to I0-day twice-daily oral linezolid for } \\
\text { ABSSSI; similar results observed when tedizolid and linezolid administered intravenously, } \\
\text { with optional oral stepdown }\end{array}$ \\
\hline $\begin{array}{l}\text { Patient-oriented evi- } \\
\text { dence }\end{array}$ & $\begin{array}{l}\text { Randomized, double- } \\
\text { blind, noninferiority clini- } \\
\text { cal trial }\end{array}$ & $\begin{array}{l}\text { Most frequent adverse events in randomised clinical trials nausea and headache;serious } \\
\text { adverse events rare; pooled data from ESTABLISH-I and ESTABLISH-2 indicated lower } \\
\text { frequency of thrombocytopenia in tedizolid-treated than linezolid-treated patients }\end{array}$ \\
\hline $\begin{array}{l}\text { Economic evidence } \\
\text { Net benefit }\end{array}$ & $\begin{array}{l}\text { High-level evidence still } \\
\text { unavailable, although } \\
\text { potential saving over line- } \\
\text { zolid for the treatment of } \\
\text { ABSSSI has been sug- } \\
\text { gested in a simulated } \\
\text { cohort }\end{array}$ & $\begin{array}{l}\text { Economic analyses warranted to optimize the use of tedizolid and maximize its advan- } \\
\text { linezolid-resistant MRSA isolates, reduced toxicity) }\end{array}$ \\
\hline
\end{tabular}

Abbreviations: ABSSI, acute bacterial skin and skin-structure infection; MRSA, methicillin-resistant Staphylococcus aureus.

The severity of ABSSSI also depends on the causative agent, with methicillin-resistant Staphylococcus aureus (MRSA) being among the most feared pathogens, not only for its well-known association with the development of invasive infection but also because of the production of Panton-Valentine leucocidin by community-acquired MRSA strains. ${ }^{5,6}$ Notably, the prevalence of MRSA among S. aureus isolates from ABSSSI may exceed 25\% in some endemic countries. ${ }^{7}$ Consequently, anti-MRSA agents are frequently an essential component of the therapeutic approach to ABSSSI.

Tedizolid phosphate (previously known as R701, DA7218), a prodrug of tedizolid (previously TR700, DA7157), is a novel oxazolidinone approved for the treatment of ABSSSI and active against MRSA. ${ }^{8,9}$ In this review, we discuss its antimicrobial and pharmacological properties, as well as the available efficacy and safety data (Table 1).

\section{Methods}

A Medline/PubMed search was conducted using various combinations of keywords and MeSH terms: "tedizolid", "TR-701", "DA-7218", "DA-7158", and "bacterial skin infection". Then, pertinent full texts, as well as abstracts and posters presented at the most recent international congresses, were evaluated and discussed, and ultimately summarized in a narrative presentation of the topic based on the highest-available level of evidence, divided into sections: mechanism of action, drug formulation and dosing, in vitro antimicrobial activity, pharmacokinetics and pharmacodynamics, efficacy in clinical studies, safety in clinical studies, potential place in therapy of tedizolid phosphate for the treatment of ABSSSI, and conclusion.

\section{Mechanism of action}

Tedizolid exerts its bacteriostatic activity by inhibiting bacteria protein synthesis through binding of the $23 \mathrm{~S}$ ribosomal RNA of the $50 \mathrm{~S}$ subunit. ${ }^{10}$ The chemical structure of tedizolid is similar to linezolid. Both are synthetic molecules containing an oxazolidinone ring (ring A) and a lateral chain at $\mathrm{C} 5$, which potentiates their activity against some Gram-positive bacteria and mycobacteria. ${ }^{11}$

The major chemical difference between the two compounds lies in the fact that tedizolid has a hydroxymethyl group in the lateral chain that is responsible for its activity against some bacterial strains with the $c f r$ gene. ${ }^{12}$ Moreover, tedizolid has a para-oriented ring structure (D-ring), which increases the number of binding sites with the peptidyl transferase center, thus enhancing its potency with respect to linezolid.

\section{Drug formulation and dosing}

Tedizolid has a double formulation for both oral and intravenous routes, which are nearly equivalent. Due to a prolonged half-life of more than 10 hours, tedizolid only needs to be administered once daily, and the recommended adult dose of tedizolid for its approved indication is 200 $\mathrm{mg}$ (regardless of the route of administration) for 6 days. No dose adjustments are required in patients with hepatic 
and/or renal impairment or in those undergoing hemodialysis. ${ }^{11}$

\section{In vitro antimicrobial activity}

Tedizolid exerts potent in vitro activity against a wide spectrum of Gram-positive bacteria, including MRSA, methicillin-resistant $S$. epidermidis, and vancomycin-resistant enterococci ${ }^{13,14}$ In a recently published study by Karlowsky et al, tedizolid displayed four fold more potent in vitro activity against $S$. aureus (both methicillin-susceptible and methicillin-resistant strains) than linezolid. ${ }^{15}$ Similarly, tedizolid and linezolid minimum inhibitory concentrations (MICs) of MRSA isolates from ABSSSIs were $0.125-0.5 \mathrm{mg}$ and $0.25-4 \mathrm{mg} / \mathrm{L}$, respectively. ${ }^{16}$ In another study involving 150 MRSA isolates, tedizolid MICs were two- to five fold lower than those of linezolid. ${ }^{17}$ Similar results were observed against enterococci, ${ }^{18}$ as also testified by another study in which the in vitro activity of tedizolid was higher than that of linezolid against 302 MRSA isolates and 220 vancomycin-resistant enterococci. ${ }^{19}$

Reduced susceptibility to tedizolid is rare, with MICs $>1$ $\mathrm{mg} / \mathrm{L}$ having been observed in only nine cases among a collection of 1,231 Gram-positive isolates. ${ }^{20}$ This possibly relies on the fact that in contrast to linezolid, tedizolid largely retains in vitro activity against Gram-positive bacteria harboring the $c f r$ gene-encoded methylase enzyme. ${ }^{21,22}$ On the other hand, chromosomal mutations in domain V of rRNA or ribosomal L3 or L4 proteins have been reported to confer resistance to both linezolid and tedizolid. ${ }^{11,23}$ An additional resistance mechanism has been found in enterococci, owing to the presence of an $\mathrm{ABC}$ transporter codified by the optrA gene carried by plasmids, which confers resistance to phenicols and oxazolidinones. ${ }^{24}$

Of note, tedizolid also displays in vitro activity against Clostridium difficile and Bacteroides fragilis. ${ }^{25,26}$ In addition, tedizolid shows more potent in vitro activity than linezolid against the most common species of Nocardia and some species of nontuberculous mycobacteria, including Mycobacterium avium complex, M. abscessus, M. fortuitum, M. marinum, M. chelonae, and M. kansasii. ${ }^{27,28}$

\section{Pharmacokinetics and pharmacodynamics}

Oral tedizolid phosphate is converted into its active moiety, tedizolid, though apical alkaline phosphatases, which allows intestinal absorption. ${ }^{29}$ Tedizolid exhibits excellent bioavailability (about 92\%), although lower values of $83 \%-86 \%$ have been reported in Chinese and Japanese patients. ${ }^{11}$ Pharmacokinetics of tedizolid after intravenous administration are similar to those following oral administration, and thus no dose adjustments are needed when switching routes. ${ }^{30}$

Tedizolid shows a high volume of distribution (67-80 $\mathrm{L}$ following a single dose, double to triple that of linezolid), and its binding to plasma proteins is $70 \%-90 \% .^{31,32}$ Tedizolid has a long half-life of 12 hours, and steady-state concentrations are achieved in 3 days. ${ }^{33,34}$ Metabolism of tedizolid occurs mostly in the liver, and the major, largely inactive metabolite isolated from feces and urine $(82 \%$ and $18 \%$, respectively) is tedizolid sulfate. ${ }^{35}$ Pharmacokinetic parameters of tedizolid are summarized in Table 2 .

With regard to special patient populations, no particular differences are observed in pharmacokinetic parameters between adults and adolescent individuals (12-17 years), while no data are available for tedizolid phosphate in subjects younger than 12 years. ${ }^{36}$ Pharmacokinetic parameters of tedizolid are also similar between nonobese and both obese (body-mass index $\geq 30 \mathrm{~kg} / \mathrm{m}^{2}$ ) and severely obese patients (body-mass index $\geq 35 \mathrm{~kg} / \mathrm{m}^{2}$ ), and thus no dosage adjustments are needed in such populations. ${ }^{37}$

Based on available murine infection-model data, tedizolid activity correlates best with AUC:MIC ratios and may be reduced in the setting of granulocytopenia. ${ }^{38}$ In a Monte Carlo simulation based on pharmacokinetic parameters in humans, the probability of reaching an $\mathrm{AUC}_{0-24}$ : MIC ratio of 3 was nearly zero for MICs $\geq 2 \mathrm{mg} / \mathrm{L}$ and $>98 \%$ for MICs $\leq 0.5 \mu \mathrm{g} / \mathrm{mL}^{11}$

Table 2 Mean (SD) tedizolid pharmacokinetic parameters

\begin{tabular}{|l|l|}
\hline & \\
\hline Dose & $200 \mathrm{mg}$ OD IV/PO \\
\hline $\mathrm{C}_{\max }(\mathrm{mg} / \mathrm{L}) \mathrm{IV}$ & $3 \pm 0.7$ \\
$\mathrm{C}_{\max }(\mathrm{mg} / \mathrm{L}) \mathbf{P O}$ & $2.2 \pm 0.7$ \\
\hline $\boldsymbol{t}_{\mathbf{1} / \mathbf{2}}$ hours & 12 \\
\hline AUC (mg*h/L) IV & $29.2 \pm 6.2$ \\
AUC (mg*h/L) PO & $25.6 \pm 8.5$ \\
\hline Protein binding & $70 \%-90 \%$ \\
\hline Excretion & Inactive metabolite \\
& $80 \%$ in feces \\
& $20 \%$ in urine \\
\hline Need for renal adjustment & No \\
\hline Need for hepatic adjustment & No \\
\hline
\end{tabular}

Abbreviations: $C_{\max }$, maximum serum concentration at steady state; $O D$, once daily, PO, per os (orally); $t_{1 / 2}$, half-life; AUC, area under the curve at steady state. 


\section{Efficacy in clinical studies}

The efficacy of tedizolid phosphate for the treatment of complicated SSSIs was initially evaluated in a phase II, double-blind, randomized trial in which the drug was administered for 5-7 days at once-daily dosages of 200 , 300, and $400 \mathrm{mg} .{ }^{39}$ The registered clinical cure rates on the test of cure in the clinically evaluable population $(n=164)$ were $98.2 \%, 94.4 \%$, and $94.4 \%$ in patients treated with daily dosages of 200,300 , and $400 \mathrm{mg}$, respectively. In patients with isolation of $S$. aureus at baseline, clinical cure rates were $96.6 \%$ overall and $96.8 \%$ when only patients with MRSA infection were considered. ${ }^{39,40}$ The approval of tedizolid phosphate for the treatment of ABSSSI by the US Food and Drug Administration in 2014 and the European Medicines Agency through the Committee for Medicinal Products for Human Use in 2015 was based on the results of two phase III randomized controlled trials: ESTABLISH-1 (NCT01170221) and ESTABLISH-2 (NCT01421511). ${ }^{8,9}$

ESTABLISH-1 was a phase III, randomized, doubleblind, noninferiority trial comparing 6-day once-daily oral tedizolid vs 10-day twice-daily oral linezolid for ABSSSI, with early clinical response at 48-72 hours as the primary outcome measure. In the intent-to-treat (ITT) population, 332 patients were randomized to receive tedizolid and 336 to receive linezolid. Noninferiority was met by with clinical response rates of $79.5 \%$ and $79.4 \%$ in the tedizolid and linezolid groups, respectively (difference $0.1 \%, 95 \%$ CI $-6.1 \%$ to $6.2 \%$, with a $10 \%$ noninferiority margin). Of note, response rates at 7-14 days after the end of treatment were similar between tedizolid-treated and linezolid-treated patients with MRSA infection $(85.2 \%$ vs $85.6 \%$, respectively). ${ }^{9}$

ESTABLISH-2 was another phase III, randomized, double-blind, noninferiority trial comparing 6-day oncedaily tedizolid vs 10-day twice-daily linezolid for ABSSI, again with early clinical response at $48-72$ hours as the primary outcome measure and a $10 \%$ noninferiority margin. However, in this study tedizolid and linezolid were administered intravenously, with optional oral stepdown. In the ITT population, 332 patients received tedizolid and 334 linezolid, with $85 \%$ and $83 \%$ rates of early clinical response, respectively (difference $2.6 \%, 95 \% \mathrm{CI}-3.0 \%$ to $8.2 \%$ ). Rates of early clinical response in patients with MRSA infection were $83 \%$ (44 of 53) and 44 of 56 (79\%) in the tedizolid and linezolid groups, respectively (difference $4.4 \%, 95 \% \mathrm{CI}-10.8 \%$ to $19.5 \%$ ). ${ }^{8}$
Some post hoc subgroup analyses have also been performed. In intravenous drug users from ESTABLISH-1 and ESTABLISH-2 (pooled ITT population), early clinical response rates were similar in the tedizolid and linezolid groups: $82.5 \%$ (151 of 183 ) and 79.6\% (164 of 206), respectively (difference $2.9 \%, 95 \% \mathrm{CI}-4.9 \%$ to $10.7 \%$ ). Similar rates of early clinical response were also observed in the tedizolid (391 of 481, 81.3\%) and linezolid (367 of $463,79.3 \%$ ) groups in non-intravenous drug users. ${ }^{41}$ Another post hoc analysis found similar efficacy of tedizolid and linezolid in outpatients from the pooled US ITT population of ESTABLISH-1 and ESTABLISH-2. Early clinical response was $84.2 \%$ and $79.0 \%$ in 403 tedizolidtreated and 410 linezolid-treated outpatients, respectively (difference $3.4 \%, 95 \% \mathrm{CI}-2.1 \%$ to $8.8 \%$ ). $^{42}$

A post hoc analysis was performed in the subgroups of patients of Latino and non-Latino origin from the pooled ITT population. In patients of Latino origin, rates of early clinical response were $80.2 \%$ (146 of 182) and $81.9 \%$ (140 of 171) for tedizolid and linezolid, respectively (difference $-1.65 \%, 95 \%$ CI $-9.88 \%$ to $6.65 \%$ ). Rates of clinical response were also similar in patients of non-Latino origin: $82.2 \%$ (396 of 482 ) for tedizolid and $78.5 \%$ (391 of 498) for linezolid (difference $3.64 \%, 95 \%$ CI $-1.37 \%$ to $8.55 \%) .{ }^{43}$ Finally, similar rates of early clinical response between tedizolid-treated and linezolid-treated patients were observed in different subgroups according to various severity measures, as well as in the subgroups of patients with and without lower-extremity infections. ${ }^{44,45}$

A phase III, randomized, controlled, open-label trial comparing tedizolid and linezolid for the treatment of ABSSSI was also conducted in Japanese patients. ${ }^{46}$ Clinical response as an exploratory end point was assessed at 7-4 days after the end of treatment. In the ITT population, clinical response rates were $77.8 \%$ (56 of 72) and $80.0 \%$ (28 of 35) in tedizolid-treated and linezolid-treated patients, respectively ( difference $-2.2 \%, 95 \% \mathrm{CI}-17.4 \%$ to $15.8 \%) .{ }^{46}$

\section{Safety in clinical studies}

The most frequent adverse events in ESTABLISH-1 and ESTABLISH-2 were nausea ( $8.5 \%$ vs $13.4 \%$ in tedizolid and linezolid groups, respectively, in ESTABLISH-1; $8 \%$ vs $11 \%$ in tedizolid and linezolid groups, respectively, in ESTABLISH-2) and headache $(6.3 \%$ vs $5.1 \%$ in tedizolid and linezolid groups, respectively, in ESTABLISH-1; 6\% vs $11 \%$ in tedizolid and linezolid groups, respectively, in ESTABLISH-2) ${ }^{8,9}$ Serious adverse events were rare in 
both studies $(1.5 \%$ vs $1.2 \%$ in tedizolid and linezolid groups, respectively, in ESTABLISH-1; $2 \%$ vs $3 \%$ in tedizolid and linezolid groups, respectively, in ESTABLISH-2). ${ }^{8,9}$

Pooled data from ESTABLISH-1 and ESTABLISH-2 indicated a lower frequency of thrombocytopenia in tedizolid-treated than in linezolid-treated patients, possibly because of the shorter treatment. ${ }^{47}$ At the posttherapy evaluation (7-14 days after the end of treatment) the platelet count was $<150,000$ cells $/ \mathrm{mm}^{3}$ in $4.2 \%$ and $7.7 \%$ of patients treated with tedizolid and linezolid, respectively (relative risk $0.55,95 \%$ CI $0.33-0.90$ ). Similar results were observed when patients with a baseline platelet count $<150,000$ were excluded from the analysis $(3.0 \%$ and $4.5 \%$ in tedizolid and linezolid groups, respectively; relative risk 0.66 , 95\% CI $0.34-1.29)^{47}$

Marketed oxazolidinones are weak, reversible inhibitors of monoamine oxidase (MAO) in vitro. ${ }^{48}$ In this regard, the available data suggest a low incidence of serotoninergic syndrome in patients treated with tedizolid, although the fact that patients treated with serotoninergic agents were excluded from ESTABLISH-1 and ESTABLISH-2 does not allow to completely exclude possible interactions. ${ }^{48,49}$ No increase in blood pressure were observed in subjects who concomitantly received tyramine or pseudoephedrine. ${ }^{48,50,51}$

Of note, the reduced inhibitory effect on central nervous system (CNS) monoamine oxidase could be due to the lower CNS penetration of tedizolid compared to linezolid, as observed in rats. ${ }^{52}$ Whether or not this could also impair the efficacy of tedizolid in some possible off-label indications (eg, CNS infections) deserves further investigation. Finally, no clinically meaningful ophthalmological or neurological alterations were observed in phase I volunteers receiving tedizolid at supratherapeutic doses for 21 days. $^{53}$

\section{Potential place in therapy of tedizolid phosphate for the treatment of ABSSSI}

In the presence of less expansive alternatives for the treatment active against MRSA, important considerations should be made to delineate the precise place in therapy for tedizolid in the treatment of ABSSSI, ultimately aiming at maximizing its cost-effectiveness by exploiting its usefulness for early switch to oral therapy and early discharge (a possible algorithm is shown in Figure 1). ${ }^{54-56} \mathrm{~A}$ first important point to be taken into account is the possibility of shorter treatment courses in comparison with other alternatives, including linezolid. In a simulated cohort of 100 inpatients, potential savings of $€ 39,348$ were projected by replacing linezolid with tedizolid for the treatment of suspected ABSSSI due to MRSA over a 1-year period. ${ }^{57,58}$ Notably, projections became cost-ineffective for simulated tedizolid treatment courses $\geq 9$ days. ${ }^{57}$

This last consideration introduces another important point worth of discussion, ie, advantages over linezolid may also be conferred by the lower toxicity observed in randomized clinical trials. However, it should be necessarily considered that this advantage, described for approved indications, such as ABSSSI (where a short 6-day course proved noninferior to 10-day linezolid), cannot be automatically extrapolated to off-label indications requiring longer tedizolid treatment courses, since long-term tolerability still has to be comprehensively assessed. Nonetheless, off-label indications certainly warrant further study, due to the undoubted theoretical advantages of less long-term toxicity than linezolid in such infections as osteomyelitis and mycobacterial diseases. In this regard, the available preliminary evidence regarding the use of tedizolid for off-label indications is briefly summarized in Table $3 .^{59-68}$ In addition, it is also true that there is no definite proof that a less expensive shorter course (6 days) of linezolid would be less effective than a 6day course of tedizolid in patients with ABSSSI. Nonetheless, attention should be paid to the fact that the available high-level evidence from randomized controlled trials refers to 6-day tedizolid and 10-day courses of linezolid therapy, and thus no firm conclusion on efficacy and safety can currently be drawn about different treatment durations than those evaluated in randomized controlled trials.

Pending dedicated postmarketing data, the potential for increased risk of oxazolidine-induced thrombocytopenia should be taken into account when treating patients with chronic liver or kidney failure. ${ }^{69}$ On the other hand, the reduced risk of drug-drug interactions with linezolid might allow the use of tedizolid concomitantly with serotoninergic or adrenergic agents in selected cases where an oxazolidinone could be considered the optimal choice (eg, possibility of step-down therapy and early discharge).

A recent systematic review and network meta-analysis compared the effectiveness of tedizolid and other antibacterial agents for treating ABSSSI caused by MRSA. ${ }^{70}$ Eligible studies were randomized controlled trials conducted in adults with complicated SSSIs or ABSSSIS caused by suspected or documented MRSA, and 15 randomized clinical trials were ultimately selected for inclusion. In fixed-effect models, tedizolid showed superior clinical response to vancomycin at the end of treatment (OR 1.7, 95\% CI 1-3), while no 


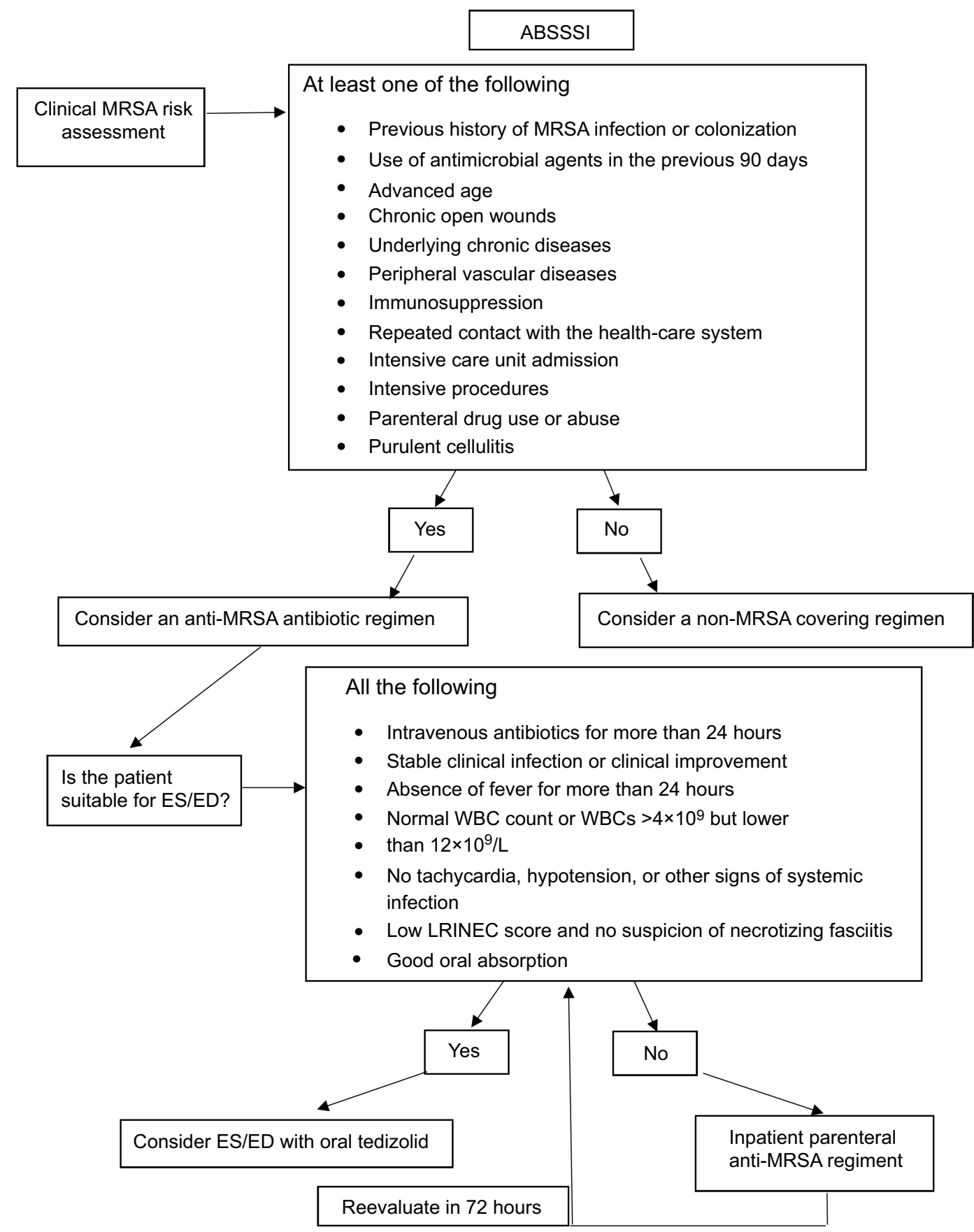

Figure I Potential algorithm for considering tedizolid-based early switch (ES) to oral therapy and early discharge (ED) in patients with ABSSI.

Note: Potential predictors of MRSA and factors indicating suitability for ES/ED have been extrapolated from the literature; data from references 54 and 55 .

Abbreviations: ABSSSI, acute bacterial skin and skin-structure infection; LRINEC, laboratory risk indicator for necrotizing fasciitis; MRSA, methicillin resistant Staphylococcus aureus; WBCs, white blood cells.

appreciable differences were observed between tedizolid and other comparators (tedizolid vs ceftaroline, OR 0.7, 95\% CI 0-30.6; tedizolid vs teicoplanin, OR 2.2, 95\% CI 0.6-9.0). Consistent results were generally observed in random-effect models. Overall, these results suggest that tedizolid is an important alternative option for the treatment of ABSSSI due to MRSA in adults, although with the inherent limitations of network meta-analyses, such as quality of included studies, limited data, and publication bias, ${ }^{71}$ and also those relying on the different types of SSSIs and outcome definitions in the different trials. Overall, it is likely that precise therapeutic algorithms developed toward a personalized, patient-oriented decision on the best anti-MRSA option on a case-by-case basis will be needed in the future, 
Table 3 Potential use of tedizolid in off-label indications and related preliminary evidence

\begin{tabular}{|c|c|}
\hline Off-label indication & Evidence \\
\hline Pneumonia & $\begin{array}{l}\text { - A noninferiority phase III, randomized, double-blind, double-dummy, controlled clinical trial } \\
\text { (NCT02019420) comparing once-daily intravenous tedizolid ( } 200 \mathrm{mg}) \text { vs twice-daily intravenous linezolid } \\
\text { (600 mg) for } 10 \text { days for the treatment of presumed Gram-positive hospital-acquired bacterial pneumonia } \\
\text { or ventilator-associated bacterial pneumonia has been recently completed, and results are awaited. } \\
\text { - In neutropenic immunocompetent mice inoculated intranasally with Staphylococcus aureus isolates, simula- } \\
\text { tion of human ELF exposure to tedizolid demonstrated greater bacterial reductions than vancomycin. }{ }^{62} \\
\text { - In a rabbit model of MRSA pneumonia, production of } \alpha \text {-toxin and Panton-Valentine leukocidin was } \\
\text { significantly inhibited when rabbits were treated with tedizolid or linezolid compared with vancomycin. }{ }^{63} \\
\text { - Tedizolid and linezolid showed antimicrobial and immunomodulatory properties in a murine model of } \\
\text { hematogenous MRSA pulmonary infection. }{ }^{61}\end{array}$ \\
\hline $\begin{array}{l}\text { Osteomyelitis and prosthetic } \\
\text { joint infections }\end{array}$ & $\begin{array}{l}\text { - A phase II, single-arm, open-label trial evaluating tolerability, safety, and efficacy of oral tedizolid for the } \\
\text { treatment of bone and joint infections is ongoing (NCT03009045). } \\
\text { - A pilot study investigating tolerance, compliance, and efficacy of tedizolid for } \geq 6 \text { weeks as monotherapy or } \\
\text { in combination for orthopedic device infections caused by Gram-positive cocci is currently recruiting } \\
\text { patients (NCT03378427). } \\
\text { - A case of successful treatment of VRE prosthetic joint infection has been reported. }{ }^{66} \\
\text { - Although inactive against biofilm-embedded S. aureus infection in the setting of bone and joint infections, } \\
\text { tedizolid seems able to prevent biofilm formation. } \\
\text { - Tedizolid alone or combined with rifampin demonstrated activity in a rat model of MRSA foreign body- } \\
\text { associated osteomyelitis, although emergence of rifampin resistance was observed. }{ }^{65}\end{array}$ \\
\hline CNS infections & $\begin{array}{l}\text { - A successful 6-month course of tedizolid therapy for CNS nocardiosis in a multiple myeloma patient has } \\
\text { been described, without additional myelotoxicity being reported. }{ }^{64}\end{array}$ \\
\hline Diabetic foot infection & $\begin{array}{l}\text { - Plasma and tissue exposure observed in in hospitalized patients with diabetic foot infections after } 200 \mathrm{mg} \\
\text { once-daily tedizolid may support further study for this indication. }{ }^{67}\end{array}$ \\
\hline Catheter-related infection & $\begin{array}{l}\text { - In a murine model of subcutaneous MRSA and MSSA catheter-related biofilm infection, a better response in } \\
\text { terms of decreased S. aureus density and bioluminescent signals after treatment with tedizolid than with } \\
\text { linezolid has been observed. } 60\end{array}$ \\
\hline Mycobacterial diseases & $\begin{array}{l}\text { - Preliminary data on long-term safety in a small sample of } 24 \text { patients have been presented in scientific } \\
\text { congresses. }{ }^{68}\end{array}$ \\
\hline
\end{tabular}

Abbreviations: CNS, central nervous system; ELF, epithelial lining fluid; MRSA, methicillin-resistant Staphylococcus aureus; MSSA, methicillin-susceptible S. aureus; VRE, vancomycin-resistant enterococci.

in order to identify precisely those patients in whom the advantages of tedizolid are maximized and those in whom the advantages of other alternatives (eg, linezolid, ceftaroline, or options allowing one-dose intravenous administration like dalbavancin or oritavancin) could be preferable.

Finally, it is necessary to mention that either sponsored or unsponsored postmarketing observational experience remains essential for pragmatically confirming effectiveness and tolerability of tedizolid outside clinical trials, both for in-label and off-label indications.

\section{Conclusion}

Optimization of management of ABSSSI should include early switch and early discharge, as well as targeting drug-resistant Gram-positive bacteria, such as MRSA. The new oxazolidinone tedizolid offers the option of intravenous to oral switch, once-daily administration, and presents multiple advantages over linezolid, including lower risk of gastrointestinal side effects, myelotoxicity, and lower risk of drugdrug interactions with compounds with serotonergic and adrenergic activity. Greater economic cost associated with this antibiotic could be offset by its shorter treatment duration and possibility of oral administration in routine clinical practice. Efforts to expand our clinical knowledge are critical to help practicing physicians determine where this drug fits into the antibiotic armamentarium.

\section{Disclosure}

MB reports grants and personal fees from Pfizer, MSD, and Cidara and personal fees from Astellas and Gilead 
outside the submitted work. DRG reports personal fees from Stepstone Pharma $\mathrm{GmbH}$ and an unconditional grant from MSD Italia outside the submitted work. The other authors report no conflicts of interest in this work.

\section{References}

1. Esposito S, Noviello S, Leone S. Epidemiology and microbiology of skin and soft tissue infections. Curr Opin Infect Dis. 2016;29(2):109115. doi:10.1097/QCO.0000000000000239

2. Garau J, Ostermann H, Medina J, et al. Current management of patients hospitalized with complicated skin and soft tissue infections across Europe (2010-2011): assessment of clinical practice patterns and reallife effectiveness of antibiotics from the REACH study. Clin Microbiol Infect. 2013;19(9):E377-E385. doi:10.1111/1469-0691.12235

3. Edelsberg J, Taneja C, Zervos M, et al. Trends in US hospital admissions for skin and soft tissue infections. Emerg Infect Dis. 2009;15(9):1516-1518. doi:10.3201/eid1509.081228

4. Pallin DJ, Egan DJ, Pelletier AJ, Espinola JA, Hooper DC, Camargo CA Jr. Increased US emergency department visits for skin and soft tissue infections, and changes in antibiotic choices, during the emergence of community-associated methicillin-resistant Staphylococcus aureus. Ann Emerg Med. 2008;51(3):291-298. doi:10.1016/j. annemergmed.2007.12.004

5. Chua K, Laurent F, Coombs G, Grayson ML, Howden BP. Antimicrobial resistance: not community-associated methicillin-resistant Staphylococcus aureus (CA-MRSA)! A clinician's guide to community MRSA - its evolving antimicrobial resistance and implications for therapy. Clin Infect Dis. 2011;52(1):99-114. doi:10.1093/cid/ciq067

6. Saeed K, Gould I, Esposito S, et al. Panton-valentine leukocidinpositive staphylococcus aureus: a position statement from the International Society of Chemotherapy. Int $J$ Antimicrob Agents. 2018;51(1):16-25. doi:10.1016/j.ijantimicag.2017.11.002

7. Bassetti M, Nicco E, Mikulska M. Why is community-associated MRSA spreading across the world and how will it change clinical practice? Int J Antimicrob Agents. 2009;34(Suppl 1):S15-S19. doi:10.1016/S0924-8579(09)70544-8

8. Moran GJ, Fang E, Corey GR, Das AF, De Anda C, Prokocimer P. Tedizolid for 6 days versus linezolid for 10 days for acute bacterial skin and skin-structure infections (ESTABLISH-2): a randomised, double-blind, phase 3, non-inferiority trial. Lancet Infect Dis. 2014;14(8):696-705. doi:10.1016/S1473-3099(14)70737-6

9. Prokocimer P, De Anda C, Fang E, Mehra P, Das A. Tedizolid phosphate vs linezolid for treatment of acute bacterial skin and skin structure infections: the ESTABLISH-1 randomized trial. Jama. 2013;309(6):559-569. doi:10.1001/jama.2013.241

10. Burdette SD, Trotman R. Tedizolid: the first once-daily oxazolidinone class antibiotic. Clin Infect Dis. 2015;61(8):1315-1321. doi:10.1093/cid/civ501

11. Ferrandez O, Urbina O, Grau S. Critical role of tedizolid in the treatment of acute bacterial skin and skin structure infections. Drug Des Devel Ther. 2017;11:65-82. doi:10.2147/DDDT.S84667

12. Locke JB, Finn J, Hilgers M, et al. Structure-activity relationships of diverse oxazolidinones for linezolid-resistant Staphylococcus aureus strains possessing the cfr methyltransferase gene or ribosomal mutations. Antimicrob Agents Chemother. 2010;54(12):5337-5343. doi:10.1128/AAC.00663-10

13. Kisgen JJ, Mansour H, Unger NR, Childs LM. Tedizolid: a new oxazolidinone antimicrobial. Am J Health Syst Pharm. 2014;71 (8):621-633. doi:10.2146/ajhp130482

14. Zhanel GG, Love R, Adam H, et al. Tedizolid: a novel oxazolidinone with potent activity against multidrug-resistant gram-positive pathogens. Drugs. 2015;75(3):253-270. doi:10.1007/s40265-015-0352-7
15. Karlowsky JA, Hackel MA, Bouchillon SK, Alder J, Sahm DF. In vitro activities of tedizolid and comparator antimicrobial agents against clinical isolates of Staphylococcus aureus collected in 12 countries from 2014 to 2016. Diagn Microbiol Infect Dis. 2017;89 (2):151-157. doi:10.1016/j.diagmicrobio.2017.07.001

16. Lee Y, Hong SK, Choi S, Im W, Yong D, Lee K. In vitro activity of tedizolid against gram-positive bacteria in patients with skin and skin structure infections and hospital-acquired pneumonia: a Korean multicenter study. Ann Lab Med. 2015;35(5):523-530. doi:10.3343/ alm.2015.35.5.523

17. Vanegas Munera JM, Ocampo Rios AM, Urrego DM, Jimenez Quiceno JN. In vitro susceptibility of methicillin-resistant Staphylococcus aureus isolates from skin and soft tissue infections to vancomycin, daptomycin, linezolid and tedizolid. Braz J Infect Dis. 2017;21(5):493-499. doi:10.1016/j.bjid.2017.03.010

18. Bai B, Hu K, Li H, et al. Effect of tedizolid on clinical Enterococcus isolates: in vitro activity, distribution of virulence factor, resistance genes and multilocus sequence typing. FEMS Microbiol Lett. 2018;365:3. doi:10.1093/femsle/fnx284

19. Barber KE, Smith JR, Raut A, Rybak MJ. Evaluation of tedizolid against Staphylococcus aureus and enterococci with reduced susceptibility to vancomycin, daptomycin or linezolid. $J$ Antimicrob Chemother. 2016;71(1):152-155. doi:10.1093/jac/dkv302

20. Bensaci M, Sahm D. Surveillance of tedizolid activity and resistance: in vitro susceptibility of gram-positive pathogens collected over 5 years from the United States and Europe. Diagn Microbiol Infect Dis. 2017;87(2):133-138. doi:10.1016/j.diagmicrobio.2016. 10.009

21. Long KS, Poehlsgaard J, Kehrenberg C, Schwarz S, Vester B. The Cfr rRNA methyltransferase confers resistance to phenicols, lincosamides, oxazolidinones, pleuromutilins, and streptogramin A antibiotics. Antimicrob Agents Chemother. 2006;50(7):2500-2505. doi:10.1128/ AAC.00131-06

22. Rybak JM, Roberts K. Tedizolid phosphate: a next-generation oxazolidinone. Infect Dis Ther. 2015. doi:10.1007/s40121-015-0060-3

23. Wilcox MH, Dmitrieva N, Gales AC, et al. Susceptibility testing and reporting of new antibiotics with a focus on tedizolid: an international working group report. Future Microbiol. 2017;12:1523-1532. doi:10.2217/fmb-2017-0106

24. Wang Y, Lv Y, Cai J, et al. A novel gene, optrA, that confers transferable resistance to oxazolidinones and phenicols and its presence in Enterococcus faecalis and Enterococcus faecium of human and animal origin. J Antimicrob Chemother. 2015;70(8):2182-2190. doi:10.1093/jac/dkv116

25. Binyamin D, Nitzan O, Azrad M, Hamo Z, Koren O, Peretz A. In vitro activity of tedizolid, dalbavancin, and ceftobiprole against clostridium difficile. Front Microbiol. 2018;9:1256. doi:10.3389/ fmicb.2018.01256

26. Goldstein EJ, Citron DM, Tyrrell KL, Leoncio ES, Merriam CV. The underappreciated in vitro activity of tedizolid against Bacteroides fragilis species, including strains resistant to metronidazole and carbapenems. Anaerobe. 2017;43:1-3. doi:10.1016/j.anaerobe.2016. 09.008

27. Brown-Elliott BA, Wallace RJ Jr. In vitro susceptibility testing of tedizolid against isolates of Nocardia. Antimicrob Agents Chemother. 2017;61:12. doi:10.1128/AAC.01537-17

28. Deshpande D, Srivastava S, Pasipanodya JG, Lee PS, Gumbo T. Tedizolid is highly bactericidal in the treatment of pulmonary Mycobacterium avium complex disease. J Antimicrob Chemother. 2017;72(suppl_2):i30-i35. doi:10.1093/jac/dkx305

29. Heimbach T, Oh DM, Li LY, et al. Absorption rate limit considerations for oral phosphate prodrugs. Pharm Res. 2003;20(6):848-856.

30. Flanagan S, Fang E, Munoz KA, Minassian SL, Prokocimer PG. Single- and multiple-dose pharmacokinetics and absolute bioavailability of tedizolid. Pharmacotherapy. 2014;34(9):891-900. doi:10.1002/phar. 1458 
31. Hall RG 2nd, Michaels HN. Profile of tedizolid phosphate and its potential in the treatment of acute bacterial skin and skin structure infections. Infect Drug Resist. 2015;8:75-82. doi:10.2147/IDR. S56691

32. Stalker DJ, Jungbluth GL. Clinical pharmacokinetics of linezolid, a novel oxazolidinone antibacterial. Clin Pharmacokinet. 2003;42 (13):1129-1140. doi:10.2165/00003088-200342130-00004

33. Flanagan SD, Bien PA, Munoz KA, Minassian SL, Prokocimer PG. Pharmacokinetics of tedizolid following oral administration: single and multiple dose, effect of food, and comparison of two solid forms of the prodrug. Pharmacotherapy. 2014;34(3):240-250. doi:10.1002/ phar. 1337

34. McBride D, Krekel T, Hsueh K, Durkin MJ. Pharmacokinetic drug evaluation of tedizolid for the treatment of skin infections. Expert Opin Drug Metab Toxicol. 2017;13(3):331-337. doi:10.1080/ 17425255.2017.1290080

35. Ong V, Flanagan S, Fang E, et al. Absorption, distribution, metabolism, and excretion of the novel antibacterial prodrug tedizolid phosphate. Drug Metab Dispos. 2014;42(8):1275-1284. doi:10.1124/ dmd.113.056697

36. Bradley JS, Flanagan SD, Arrieta AC, Jacobs R, Capparelli E, Prokocimer P. Pharmacokinetics, safety and tolerability of single oral or intravenous administration of $200 \mathrm{mg}$ tedizolid phosphate in adolescents. Pediatr Infect Dis J. 2016;35(6):628-633. doi:10.1097/ INF.0000000000001096

37. Pai MP. Pharmacokinetics of tedizolid in morbidly obese and covariate-matched nonobese adults. Antimicrob Agents Chemother. 2016;60 (8):4585-4589. doi:10.1128/AAC.00682-16

38. Lodise TP, Drusano GL. Use of pharmacokinetic/pharmacodynamic systems analyses to inform dose selection of tedizolid phosphate. Clin Infect Dis. 2014;58(Suppl 1):S28-S34. doi:10.1093/cid/cit615

39. Prokocimer P, Bien P, Surber J, et al. Phase 2, randomized, doubleblind, dose-ranging study evaluating the safety, tolerability, population pharmacokinetics, and efficacy of oral torezolid phosphate in patients with complicated skin and skin structure infections. Antimicrob Agents Chemother. 2011;55(2):583-592. doi:10.1128/ AAC.00076-10

40. Prokocimer P, Bien P, Deanda C, Pillar CM, Bartizal K. In vitro activity and microbiological efficacy of tedizolid (TR-700) against Gram-positive clinical isolates from a phase 2 study of oral tedizolid phosphate (TR-701) in patients with complicated skin and skin structure infections. Antimicrob Agents Chemother. 2012;56(9):46084613. doi:10.1128/AAC.00458-12

41. Moran GJ, De Anda C, Das AF, Green S, Mehra P, Efficacy PP. Safety of tedizolid and linezolid for the treatment of acute bacterial skin and skin structure infections in injection drug users: analysis of two clinical trials. Infect Dis Ther. 2018;7(4):509-522. doi:10.1007/ s40121-018-0211-4

42. De Anda C, Anuskiewicz S, Prokocimer P, Vazquez J. Outpatient treatment of acute bacterial skin and skin structure infections (ABSSSI) with tedizolid phosphate and linezolid in patients in the United States: subgroup analysis of 2 randomized phase 3 trials. Medicine (Baltimore). 2017;96(52):e9163. doi:10.1097/MD.0000 000000009163

43. Ortiz-Covarrubias A, Fang E, Prokocimer PG, et al. Efficacy, safety, tolerability and population pharmacokinetics of tedizolid, a novel antibiotic, in Latino patients with acute bacterial skin and skin structure infections. Braz J Infect Dis. 2016;20(2):184-192. doi:10.1016/j. bjid.2015.12.007

44. Joseph WS, Culshaw D, Anuskiewicz S, De Anda C, Prokocimer P. Tedizolid and linezolid for treatment of acute bacterial skin and skin structure infections of the lower extremity versus nonlower-extremity infectionspooled analysis of two phase 3 trials. $J$ Am Podiatr Med Assoc. 2017;107(4):264-271. doi:10.7547/15218
45. Sandison T, De Anda C, Fang E, Das AF, Prokocimer P. Clinical response of tedizolid versus linezolid in acute bacterial skin and skin structure infections by severity measure using a pooled analysis from two phase 3 double-blind trials. Antimicrob Agents Chemother. 2017;61:5. doi:10.1128/AAC.02687-16

46. Mikamo H, Takesue Y, Iwamoto Y, et al. Efficacy, safety and pharmacokinetics of tedizolid versus linezolid in patients with skin and soft tissue infections in Japan - Results of a randomised, multicentre phase 3 study. J Infect Chemother. 2018;24(6):434-442. doi:10.1016/ j.jiac.2018.01.010

47. Lodise TP, Fang E, Minassian SL, Prokocimer PG. Platelet profile in patients with acute bacterial skin and skin structure infections receiving tedizolid or linezolid: findings from the Phase 3 ESTABLISH clinical trials. Antimicrob Agents Chemother. 2014;58(12):71987204. doi:10.1128/AAC.03509-14

48. Flanagan S, Bartizal K, Minassian SL, Fang E, Prokocimer P. In vitro, in vivo, and clinical studies of tedizolid to assess the potential for peripheral or central monoamine oxidase interactions. Antimicrob Agents Chemother. 2013;57(7):3060-3066. doi:10.1128/AAC.0043113

49. Hall RG 2nd, Smith WJ, Putnam WC, Pass SE. An evaluation of tedizolid for the treatment of MRSA infections. Expert Opin Pharmacother. 2018;19(13):1489-1494. doi:10.1080/146565662018. 1519021

50. Das D, Tulkens PM, Mehra P, Fang E, Prokocimer P. Tedizolid phosphate for the management of acute bacterial skin and skin structure infections: safety summary. Clin Infect Dis. 2014;58(Suppl 1):S51-S57. doi:10.1093/cid/cit618

51. Flanagan S, Minassian SL, Prokocimer P. Pharmacokinetics of tedizolid and pseudoephedrine administered alone or in combination in healthy volunteers. J Clin Med. 2018;7:6. doi:10.3390/jcm7060150

52. European Medicines Agency. SIVEXTRO®. Assessment report as adopted by the CHMP with all information of a commercially confidential nature deleted. Available from: http://www.ema.europa.eu/ docs/en_GB/document_library/EPAR_-_Public_assessment_report/ human/002846/WC500184803.pdf. Accessed May 22, 2019.

53. Fang E, Munoz KA, Prokocimer P. Characterization of neurologic and ophthalmologic safety of oral administration of tedizolid for up to 21 days in healthy volunteers. Am J Ther. 2017;24(2):e227-e233. doi:10.1097/MJT.0000000000000534

54. Bassetti M, Baguneid M, Bouza E, Dryden M, Nathwani D, Wilcox M. European perspective and update on the management of complicated skin and soft tissue infections due to methicillin-resistant Staphylococcus aureus after more than 10 years of experience with linezolid. Clin Microbiol Infect. 2014;20(Suppl 4):3-18. doi:10.1111/ 1469-0691.12463

55. Bassetti M, Eckmann C, Peghin M, Carnelutti A, Righi E. When to switch to an oral treatment and/or to discharge a patient with skin and soft tissue infections. Curr Opin Infect Dis. 2018;31(2):163-169. doi:10.1097/QCO.0000000000000434

56. Wong $\mathrm{CH}$, Khin LW, Heng KS, Tan KC, Low CO. The LRINEC (Laboratory risk indicator for necrotizing fasciitis) score: a tool for distinguishing necrotizing fasciitis from other soft tissue infections. Crit Care Med. 2004;32(7):1535-1541.

57. Belkova YA, Rachina SA, Kozlov RS, Golub AV, Portnyagina US, Shamaeva SH. Cost implications of tedizolid introduction for the treatment of complicated skin and soft tissue infections in a Russian multifield hospital. Value Health. 2017;20(9):A782. doi:10.1016/j. jval.2017.08.2277

58. Bouza E, Munoz P, Burillo A. The role of tedizolid in skin and soft tissue infections. Curr Opin Infect Dis. 2018;31(2):131-140. doi:10.1097/QCO.0000000000000439

59. Abad L, Tafani V, Tasse J, et al. Evaluation of the ability of linezolid and tedizolid to eradicate intraosteoblastic and biofilm-embedded Staphylococcus aureus in the bone and joint infection setting. $J$ Antimicrob Chemother. 2019;74:625-632. 
60. Bayer AS, Abdelhady W, Li L, Gonzales R, Xiong YQ. Comparative efficacies of tedizolid phosphate, linezolid, and vancomycin in a murine model of subcutaneous catheter-related biofilm infection due to methicillin-susceptible and -resistant Staphylococcus aureus. Antimicrob Agents Chemother. 2016;60(8):5092-5096. doi:10.1128/AAC.00880-16

61. Kaku N, Morinaga Y, Takeda K, et al. Antimicrobial and immunomodulatory effects of tedizolid against methicillin-resistant Staphylococcus aureus in a murine model of hematogenous pulmonary infection. Int $J$ Med Microbiol. 2016;306(6):421-428. doi:10.1016/j.ijmm.2016.05.010

62. Kidd JM, Abdelraouf K, Nicolau DP. Comparative efficacy of humansimulated epithelial lining fluid exposures of tedizolid, linezolid and vancomycin in neutropenic and immunocompetent murine models of staphylococcal pneumonia. J Antimicrob Chemother. 2019;74:970-977.

63. Le VT, Le HN, Pinheiro MG, et al. Effects of tedizolid phosphate on survival outcomes and suppression of production of staphylococcal toxins in a rabbit model of methicillin-resistant Staphylococcus aureus necrotizing pneumonia. Antimicrob Agents Chemother. 2017;61:4. doi:10.1128/AAC.02734-16

64. Matin A, Sharma S, Mathur P, Apewokin SK. Myelosuppressionsparing treatment of central nervous system nocardiosis in a multiple myeloma patient utilizing a tedizolid-based regimen: a case report. Int $J$ Antimicrob Agents. 2017;49(4):488-492. doi:10.1016/j. ijantimicag.2016.11.032

65. Park KH, Greenwood-Quaintance KE, Mandrekar J, Patel R. Activity of tedizolid in methicillin-resistant Staphylococcus aureus experimental foreign body-associated osteomyelitis. Antimicrob Agents Chemother. 2016;60(11):6568-6572. doi:10.1128/AAC.01248-16
66. Si S, Durkin MJ, Mercier MM, Yarbrough ML, Liang SY. Successful treatment of prosthetic joint infection due to vancomycin-resistant enterococci with tedizolid. Infect Dis Clin Pract (Baltim Md). 2017;25(2):105-107. doi:10.1097/IPC.0000000000000469

67. Stainton SM, Monogue ML, Baummer-Carr A, et al. Comparative assessment of tedizolid pharmacokinetics and tissue penetration between diabetic patients with wound infections and healthy volunteers via in vivo microdialysis. Antimicrob Agents Chemother. 2018;62:1.

68. Kim T, Wills A, Markus A, Prevots DR, Olivier KN. Safety and tolerability of long term use of tedizolid for treatment of nontuberculous mycobacterial infections. Open Forum Infect Dis. 2016;3 (suppl 1):577. doi:10.1093/ofid/ofw172.440

69. Nukui Y, Hatakeyama S, Okamoto K, et al. High plasma linezolid concentration and impaired renal function affect development of linezolid-induced thrombocytopenia. $J$ Antimicrob Chemother. 2013;68(9):2128-2133. doi:10.1093/jac/dkt133

70. McCool R, Gould IM, Eales J, et al. Systematic review and network meta-analysis of tedizolid for the treatment of acute bacterial skin and skin structure infections caused by MRSA. BMC Infect Dis. 2017;17 (1):39. doi:10.1186/s12879-017-2757-2

71. Cipriani A, Higgins JP, Geddes JR, Salanti G. Conceptual and technical challenges in network meta-analysis. Ann Intern Med. 2013;159 (2):130-137. doi:10.7326/0003-4819-159-2-201307160-00008
Core Evidence

\section{Publish your work in this journal}

Core Evidence is an international, peer-reviewed open-access journal evaluating the evidence underlying the potential place in therapy of drugs throughout their development lifecycle from preclinical to post launch. The focus of each review is to evaluate the case for a new drug or class in outcome terms in specific indications and patient groups.

Submit your manuscript here: https://www.dovepress.com/core-evidence-journal

The manuscript management system is completely online and includes a very quick and fair peer-review system, which is all easy to use. Visit http://www.dovepress.com/testimonials.php to read real quotes from published authors. 